\title{
Analysis and Measurement of Polarization Conversion in a Periodically Loaded Dielectric Waveguide
}

\author{
Takashi Ando, Student Member, IEEE, Toshiyuki Murata, Hiroki Nakayama, Junji Yamauchi, Member, IEEE, and \\ Hisamatsu Nakano, Fellow, IEEE
}

\begin{abstract}
The full-vectorial beam-propagation method with the improved finite-difference formula is applied to the analysis of a TE/TM mode converter based on an asymmetric periodic loaded waveguide. The polarization conversion behavior is measured at a microwave frequency. It is demonstrated that the calculated complete conversion length is in agreement with the experimental result.
\end{abstract}

Index Terms-Dielectric waveguides, FDTD methods, finite-difference methods, loaded waveguides, optical waveguides, polarization.

\section{INTRODUCTION}

$\mathbf{T}$ E/TM mode converters are essential for polarization diversity receivers in coherent optical systems and for sensor applications. Shani et al. [1] proposed passive converters based on asymmetric periodic-loaded rib waveguides and experimentally demonstrated polarization rotation in the waveguides. The operation principle of the polarization rotation was explained by the coupled-mode theory based on scalar modes [2]. For the complete design of the loaded waveguides, full-vectorial simulations [3]-[5] are required.

So far, the full-vectorial beam-propagation method (VBPM) has often been utilized for the evaluations of polarization-conversion properties in asymmetric periodic-loaded rib waveguides [6]-[9]. However, the simulated complete conversion lengths do not necessarily agree with experimental results. This is probably due to the difficulties in accurately fabricating and measuring devices at optical frequencies. It follows that the effectiveness of the VBPM has not completely been proved in the analysis of practical polarization-dependent devices.

We should notice that the devices can geometrically be expanded on the basis of a frequency transformation from optical to microwave frequencies [10]. The geometric expansion leads to decreases in the sensitivities to fabrication and measurement errors. Hence, the experimental investigation at microwave frequencies must be suitable for the confirmation of the validity of numerical methods developed for an optical waveguide analysis.

In this letter, polarization conversion in a periodically loaded waveguide is numerically and experimentally investigated at a

Manuscript received March 19, 2002; revised May 14, 2002.

The authors are with Faculty of Engineering, Hosei University, Koganei,

Tokyo 184-8584, Japan.

Publisher Item Identifier 10.1109/LPT.2002.801097.

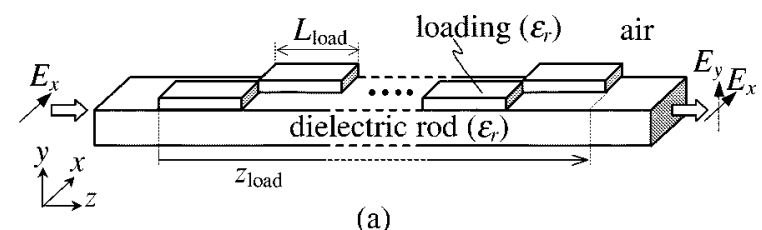

(a)

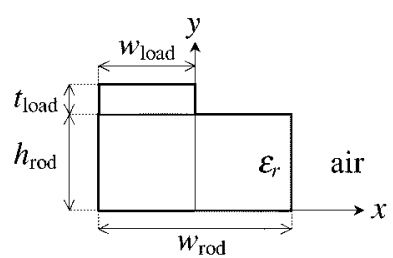

(b)

Fig. 1. Configuration $\left(\epsilon_{r}=2.8, w_{\text {rod }}=13 \mathrm{~mm}, h_{\text {rod }}=6.5 \mathrm{~mm}, w_{\text {load }}=\right.$ $6.5 \mathrm{~mm}$ ). (a) Overall geometry. (b) Cross section.

microwave frequency of $f=15 \mathrm{GHz}$. The VBPM with the improved finite-difference formula (IFD-VBPM) [11] is used for the analysis of propagating field along the waveguide. It is revealed that the polarization-conversion behavior evaluated by the IFD-VBPM agrees well with the experimental result. This letter also shows that the results comparable to the IFD-VBPM are obtained from the finite-difference time-domain (FDTD) method [12].

\section{CONFIGURATION AND EXPERIMENTAL SETUP}

Fig. 1 shows the configuration of a periodically loaded waveguide, which is simply composed of a dielectric rod and loadings. Note that the simple structure is adequate to evaluate the essential property of a periodically loaded waveguide, although a superior structure with angled facets [7], [8] has been proposed.

The rod and loadings are made of a polycarbonate of $\epsilon_{r}=$ 2.8. The dimensions of the cross section of the rod are fixed to be $w_{\text {rod }} \times h_{\text {rod }}=13 \mathrm{~mm} \times 6.5 \mathrm{~mm}$ so that the rod operates as a single-mode waveguide at a testing frequency of $f=15 \mathrm{GHz}(\lambda=20 \mathrm{~mm})$.

The width of the loading is typically chosen to be half of $w_{\text {rod }}$, i.e., $w_{\text {load }}=6.5 \mathrm{~mm}$. The thickness of the loading is designated as $t_{\text {load }}$, which is taken to be two values $\left(t_{\text {load }}=2 \mathrm{~mm}\right.$ and 3 $\mathrm{mm}$ ) to investigate the effect of $t_{\text {load }}$ on polarization conversion length. The loading length $L_{\text {load }}$ is determined by the phase matching condition between the fundamental TE- and TM-like modes in the loading section, as will be shown in Section III. 


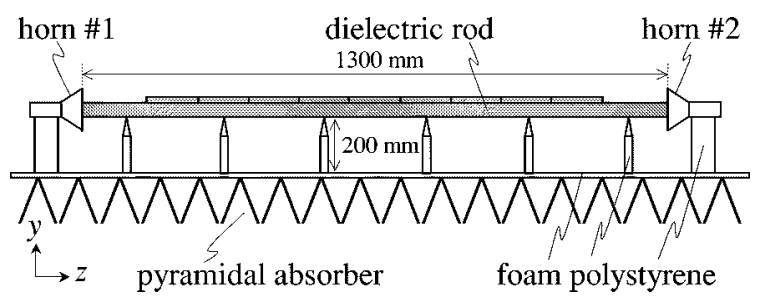

Fig. 2. Experimental setup for measuring polarization-conversion property.

The polarization conversion in the waveguide is measured using the experimental setup illustrated in Fig. 2. The rod is supported by foam-polystyrene pillars and is fed by the horn launcher \#1 through a metallic waveguide (WR-51). The dimensions of the horn aperture are $49 \times 66 \mathrm{~mm}$. When the metallic waveguide is excited with the fundamental mode whose major electric field component is $E_{x}$, part of the power radiating from the horn is converted into the unguided mode power and the remaining power into the $E_{11}^{x}$ mode power [13] (the ratio of the $E_{11}^{x}$ mode power to the total radiation power is evaluated to be greater than $80 \%$ by a preliminary calculation). The loadings with a length of $L_{\text {load }} / 2$ are set on the rod one after another, for the evaluation of the conversion behavior as a function of the overall length of the loading sections. The guided waves in the $x$ and $y$ polarizations are alternately received by the horn $\# 2$. The measured power of $E_{x}$ is regarded as the $E_{11}^{x}$ (TE) mode power and that of $E_{y}$ as the $E_{11}^{y}(\mathrm{TM})$ mode power.

\section{DISCUSSION}

The IFD-VBPM formulated by the two transverse electric field components [11] is applied to the design and evaluation of the waveguide. The waveguide is assumed to be linear lossless medium in the calculation. The rod is excited with the $E_{11}^{x}$ mode whose field profiles are obtained by the imaginary distance scheme [11], [14]. The computational parameters are fixed to be $\Delta x=\Delta y=0.5 \mathrm{~mm}$ and $\Delta z=1 \mathrm{~mm}$. The number of transverse grid points is $L_{x} \times L_{y}=150 \times 150$. The perfectly matched layer is employed for absorbing outgoing waves at the computational edges.

As mentioned previously, the knowledge of the eigenmodes is of fundamental importance in the design of the loading section. $L_{\text {load }}$ is taken to be half of the beat length between the fundamental TE- and TM-like modes in the loading section, i.e., $L_{\text {load }}=\pi /\left(\beta_{\mathrm{TE}}-\beta_{\mathrm{TM}}\right)$, where $\beta_{\mathrm{TE}}$ and $\beta_{\mathrm{TM}}$ are the propagation constants of the TE- and TM-like modes. $\beta_{\mathrm{TE}}$ and $\beta_{\mathrm{TM}}$ can be obtained using the imaginary distance scheme. As a result, the loading lengths for $t_{\text {load }}=2$ and $3 \mathrm{~mm}$ are calculated to be $L_{\text {load }}=127$ and $162 \mathrm{~mm}$, respectively.

The constructive interference between the TE- and TM-like modes in the loading sections will lead to a complete polarization conversion. The converted power is given by $P_{c}=\sin ^{2}\left(\kappa L_{\text {load }} N\right)$ [7, eq. (1)], where $\kappa$ is a coupling coefficient and $N$ is the number of loadings. When $N L_{\text {load }}$ is equal to $\pi / 2|\kappa|$, the complete conversion can be obtained. Auxiliary calculations showed that the values of $|\kappa|$ for $t_{\text {load }}=2$ and $3 \mathrm{~mm}$ are approximately $2.5 \times 10^{-3}$ and $3.1 \times 10^{-3}$, respectively. Consequently, it is expected that the excited $E_{11}^{x}$ (TE)

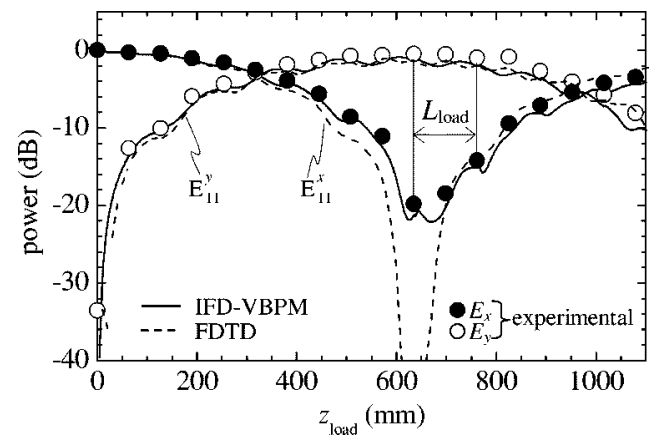

Fig. 3. Guided mode power for $t_{\text {load }}=2 \mathrm{~mm}$ as a function of $z_{\text {load }}\left(L_{\text {load }}=\right.$ $127 \mathrm{~mm})$.

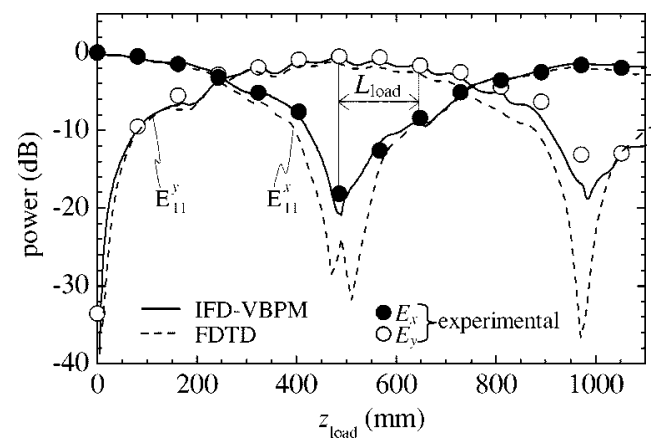

Fig. 4. Guided mode power for $t_{\text {load }}=3 \mathrm{~mm}$ as a function of $z_{\text {load }}\left(L_{\text {load }}=\right.$ $162 \mathrm{~mm}$ ).

mode is almost converted into the $E_{11}^{y}$ (TM) mode at $N=5$ for $t_{\text {load }}=2 \mathrm{~mm}$, and at $N=3$ for $t_{\text {load }}=3 \mathrm{~mm}$.

Fig. 3 shows the guided mode power for $t_{\text {load }}=2 \mathrm{~mm}$ as a function of $z_{\text {load }}$. The $z_{\text {load }}$ is defined as the length, where the loadings are set on the rod (see Fig. 1). The power is normalized to the excited $E_{11}^{x}$ mode power. The data calculated by the IFD-VBPM are presented by solid lines. As expected, almost complete conversion can be obtained at $z_{\text {load }}=635 \mathrm{~mm}(=$ $\left.5 L_{\text {load }}\right)$. The calculated conversion behavior agrees well with the experimental data plotted by solid and open circles.

Fig. 4 shows the conversion property for $t_{\text {load }}=3 \mathrm{~mm}$. Good agreement is again found between the numerical and experimental results. The almost complete conversion length is decreased from 635 to $486 \mathrm{~mm}\left(=3 L_{\text {load }}\right)$, when $t_{\text {load }}$ is changed from 2 to $3 \mathrm{~mm}$.

Although not presented, we numerically and experimentally confirmed that properties similar to those in Figs. 3 and 4 are obtained for the TM-to-TE ( $E_{11}^{y}$-to- $E_{11}^{x}$ ) mode conversion.

Note that reflected waves should be generated at the interface between the loading sections. Unfortunately, the IFD-VBPM assumes only forward traveling waves, so that the reflected waves are neglected. To investigate the influence of the reflected waves on the conversion behavior, we also analyze the waveguide by the FDTD method that is applicable to treating reflection problems.

The values obtained by the FDTD analysis are plotted by dotted lines in Figs. 3 and 4. As can be seen, the conversion behavior evaluated by the FDTD method substantially agrees with that by the IFD-VBPM. The overall loss during the complete conversion process (including scattering and reflection losses) is calculated to be approximately $1 \mathrm{~dB}$ for $t_{\text {load }}=2 \mathrm{~mm}$. Note that 
the discrepancy between the IFD-VBPM and the FDTD method is observed for low-power levels of less than $-20 \mathrm{~dB}$. This is because the propagating minor field for the IFD-VBPM is slightly deformed by numerical artifacts, which may be caused by the negligence of the reflected waves and by the use of the slowly varying envelope approximation.

As is well known, the IFD-VBPM requires less computational time and memories in comparison with the FDTD method. The calculations have been performed on a PC with Pentium 1-GHz processor and 2-GB memories. For example, we need approximately 7 days and $1.9 \mathrm{~GB}$ memories in the FDTD analysis to obtain the data for $z_{\text {load }}=1100 \mathrm{~mm}$. On the other hand, the IFD-VBPM requires approximately $10 \mathrm{~min}$ and $70 \mathrm{MB}$. It is concluded that the IFD-VBPM enables us to efficiently evaluate polarization-dependent devices, provided that reflected waves are negligible.

\section{CONCLUSION}

Polarization conversion in a periodically loaded dielectric waveguide has been investigated numerically and experimentally at a microwave frequency. The IFD-VBPM is used for the analysis of propagating field along the waveguide. The calculated complete conversion length agrees well with the experimental result. It is demonstrated that the IFD-VBPM is powerful tool of analyzing practical polarization-dependent devices.

\section{ACKNOWLEDGMENT}

The authors would like to thank N. Agari for his basic investigations of a periodically loaded optical waveguide.

\section{REFERENCES}

[1] Y. Shani, R. Alferness, T. Koch, U. Koren, M. Oron, B. I. Miller, and M. G. Young, "Polarization rotation in asymmetric periodic loaded rib waveguides," Appl. Phys. Lett., vol. 59, no. 11, pp. 1278-1280, 1991.

[2] W. P. Huang and Z. M. Mao, "Polarization rotation in periodic loaded rib waveguides," J. Lightwave Technol., vol. 10, pp. 1825-1831, Dec. 1992.

[3] C. M. Weinert and H. Heidrich, "Vectorial simulation of passive TE/TM mode converter devices on InP," IEEE Photon. Technol. Lett., vol. 5, pp. 324-326, Mar. 1993.

[4] F. J. Mustieles, E. Ballesteros, and F. H. Gil, "Multimodal analysis method for the design of passive TE/TM converters in integrated waveguides," IEEE Photon. Technol. Lett., vol. 5, pp. 809-811, July 1993.

[5] K. Mertens, B. Scholl, and H. J. Schmitt, "Strong polarization conversion in periodically loaded strip waveguides," IEEE Photon. Technol. Lett., vol. 10, pp. 1133-1135, Aug. 1998.

[6] W. P. Huang and C. L. Xu, "Simulation of three-dimensional optical waveguides by a full-vector beam propagation method," IEEE $J$. Quantum Electron., vol. 29, pp. 2639-2649, Oct. 1993.

[7] J. J. G. M. van der Tol, F. Hakimzadeh, J. W. Pedersen, D. Li, and H. van Brug, "A new short and low-loss passive polarization converter on InP,” IEEE Photon. Technol. Lett., vol. 7, pp. 32-34, Jan. 1995.

[8] D. Li, H. van Brug, and H. J. Frankena, "Application of a fully vectorial beam propagation method," Opt. Quantum Electron., vol. 29, no. 1, pp. 313-322, 1997.

[9] S. S. A. Obayya, B. M. A. Rahman, and H. A. E. Mikati, "Vector beam propagation analysis of polarization conversion in periodically loaded waveguides," IEEE Photon. Technol. Lett., vol. 12, pp. 1346-1348, Oct. 2000.

[10] J. Albrecht and E.-G. Neumann, "Simulation of the near field of single-mode fibers by means of a microwave model," Microwaves, Optics Acoust., vol. 3, no. 3, pp. 109-114, 1979.

[11] J. Yamauchi, G. Takahashi, and H. Nakano, "Full-vectorial beam-propagation method based on the McKee-Mitchell scheme with improved finite-difference formulas," J. Lightwave Technol., vol. 16, pp. 2458-2464, Dec. 1998.

[12] A. Taflove and S. C. Hagness, Computational Electrodynamics, the Finite-Difference Time-Domain Method, second ed. New York: Artech, 2000.

[13] T. Ando, J. Yamauchi, and H. Nakano, "Rectangular dielectric-rod fed by a metallic waveguide," Proc. IEE-Microw. Antennas Propagat., vol. 149, pp. 92-97, Apr. 2002.

[14] D. Yevick and W. Bardyszewski, "Correspondence of variation finitedifference (relaxation) and imaginary-distance propagation methods for modal analysis," Opt. Lett., vol. 17, no. 5, pp. 329-330, 1992. 